\title{
A Promising Scheme for Portfolio Selection to Gain Pragmatic Pool-based Electricity Market Returns under Uncertain Circumstances
}

\author{
Waqas Ahmad WATTOO ${ }^{1,2 *}$, Donghan FENG ${ }^{1}$, Muhammad YOUSIF ${ }^{1}$, Sohaib TAHIR ${ }^{2}$ \\ ${ }^{1}$ Department of Electrical Engineering, SEIEE, Shanghai Jiaotong University, Minhang District, \\ Shanghai, China \\ vickz88@sjtu.edu.cn (*Correspondingauthor), seed@sjtu.edu.cn, yousif@sjtu.edu.cn \\ ${ }^{2}$ Department of Electrical Engineering, COMSATS University Islamabad (CUI), Sahiwal Campus, \\ Sahiwal, Pakistan \\ sohaibchauhdary@hotmail.com
}

\begin{abstract}
The prospective trading position of an independent power production company (IPPCo) is fortified by handling its portfolio between numerous trading preferences. Owing to uncertain circumstances, the future profits of these trading preferences are estimated by applying fuzzy or probabilistic methods. Conventional schemes are usually found inappropriate in computing the uncertainty and revealing the deviation between apparent and actual market profits. Based on this deviation, a non-probabilistic Information Gap Decision (IGD) theory is applied in this paper to present the optimum scheme for IPPCo's portfolio selection. The performance of IPPCo is modeled and critically analyzed in determining its trading scheme. Numerous decisions are validated for IPPCo through various criteria in results to plump for the best outcome. The risk averting behavior of IPPCo's framework leads to offer robust decisions towards losses, whereas, its risk captivating behavior provides the facility to acquire windfall gain. The presented scheme is also corroborated through real time scenarios of PJM market.
\end{abstract}

Keywords: Uncertain market returns, Independent power production company (IPPCo), Pool electricity market, Portfolio optimization, Information gap decision (IGD) theory.

\section{Introduction}

The deregulated power sector has acquainted with numerous markets and alternate options for trading, as a result various options become available to independent power companies (IPCs) for trading electricity. Advanced decisions are made by these companies in the electricity markets (Đogić, 2017). In real-time situations, several unpredictable factors affect the prices of electricity, which results in high market uncertainty (Orgaz et al., 2018). Owing to this uncertainty in the markets, the contracts and agreements in the electricity markets also gets uncertain, which leads to price-volatility in for each contract. The risk reduction and profit maximization are the prime preferences of the power generation companies (IPPCos). The aforementioned preferences can be achieved by using the multiple trading choices (Dagoumas et al., 2017). This formulated allocation of energy to multiple contracts by deliberating the risk preference of a corporation and risk-profit tradeoff in a potential market is termed as portfolio optimization (Faia et al., 2018).

Owing to development in the markets and plummeting the relative risks, IPPs are suggested to inflate their generation and sales portfolios with various renewable and conventional alternatives.
This strategy is illustrious and quite familiar for the researchers, as this can be noticed from the perspective of classical portfolio theory. The addition of new securities in the market may lead to a drop in the portfolio risk, as revealed in classical portfolio theory. The applications of Classical portfolio theory cannot only be understood through investigations applied over the stock markets but it can also be proved through mathematical equations and computations (Gökgöz \& Atmaca, 2012, Statman, 1987). Though, this theory isn't adequate to be employed in copious securities as it isn't a systematic approach to reduce risk (variance) (Markowitz, 1952). The finest way is to avoid investing in securities with high correlation factors. Based on trading protocols, the competitive electricity markets essentially consist of energy market (day-ahead, hour-ahead, and real-time balancing market) and several contractual instruments, such as forward and future contracts (Shahidehpour et al., 2003).

Power producers and end retailers are trying to employ the bilateral contracts, options and futures to minimize spot markets risks (Gedra, 1994, Menniti et al.). However, in a competitive market, every power producer's main goal is to 
maximize its profit and minimize the associated risks, and it needs the clear determination of these risks as well as necessary actions should be taken in order to achieve the said factors. Likelihood of suffering from damage or harm: threat or danger is called risk. Uncertainty cause risk. But uncertainty and risk are two different factors: something which can controlled is called risk while something which is beyond anyone's control can be said as uncertainty.

To take right decisions, numerous existing strategies exploited the estimation of future conditions and used fuzzy membership functions or probability functions (Carlsson et al., 2003). Historical data is frequently employed to figure out the estimations for taking right decision. However, factor of unreliability is relatively higher in approximating the future profits based on the past profits, and uncertainty is quantified by using probability distribution function. Portfolio selection involves various uncertain trading opportunities. Precise forecast of numerous input parameters is considered as a prerequisite for optimal portfolio selection e.g. variance, individual profit and correlation between several uncertain options. During decision making, precise forecasts are not available and due to erroneous estimations, it results in incongruous diversification. This results in serious losses owing to involvement of large quantum of power. In addition to that, most of the approaches suggest decisions by taking into account the risk-averse nature of investors. The portfolio selection decisions are based on risk-profit trade-off. However, practical market scenario necessitates broader criteria to be considered for improving portfolio performance that meets IPPCo's aspirations.

If existing portfolio selection strategies are compared, IGD theory provides verdicts, which are reliable and they are grounded on reliant information gap between real and projected values, without relying on the estimates. This theory helps in producing the portfolios which shows robustness against losses and own opportunistic ability to capture windfall gains (Ben-Haim, 2001, Zhang et al., 2018). IGD theory becomes an attractive option to solve and understand variety of market issues due to huge volatility in electricity markets (Aien et al., 2016). These issues are optimal bidding, IPPCo's self- scheduling, bidding for electricity acquisition and favorable planning for regular clients (He et al., 2016, Jalilvand-Nejad et al., 2017, Nojavan et al., 2015a, Nojavan et al., 2015b, Vatani et al., 2018).

In this paper, portfolio selection is critically considered and a quantitative scheme is presented on the basis of IGD theory to cope with severe uncertainties. The assets with uncertain profits are also taken into account while proposing the presented scheme. Price uncertainty of pool and congestion charges are analytically deliberated during trading of electricity by IPPCo in spot and contract markets. The proposed formulation deals with uncertainty of each trading alternative and their corresponding counterparts. Correspondingly, the real-time data from the PJM market is employed to validate the authenticity of the proposed scheme. The study indicates that there exists numerous trade-offs between robustness and reward, windfall gain in consort with opportunities, and robustness as well as opportuneness, for selecting a portfolio.

\section{Agenda of IGD Theory}

IGD theory is a compulsive theory for making good decisions in case that inadequate information about the input parameters is available. The theory is found robust against failures and facilitates in capturing windfall gains. Significant data is used in this technique to calculate approximations of relative degree of deviation for uncertain asset profits in selecting a portfolio. Though, if there exists a noticeable deviation among actual and estimated values of these parameters. IGD theory considers the difference between actual and estimated values as a regulated parameter and deals with it as an unconstrained parameter (Ben-Haim, 2006).

IGD thoery evaluates decisions at many points, as uncertainty varies from estimation in an unbounded manner. These points are different values of uncertainty parameter evaluated from different performance requirements. To facilitate decision maker in comparing several trading decisions that meet the standards for system performance, these points are depleted which are actually distinct values of uncertainty parameters evaluated according to the performance

http://www.sic.ici.ro 
requirements. Three fundamental models are exploited to address the problems of IGD theory during decisions making:

\subsection{Description for System Modelling}

Decisions are taken on the basis of the system model $E(v, u)$ which states input/output configuration of system, where, $v$ depicts the set of decision variables and $u$ represents the uncertainty in a parameter (Ben-Haim, 2006). The parameter which deviates in an unidentified transient way from its nominal approximation is input parameter of interest, $u$. Objective function is the system model i.e. portfolio profit acquired during available trading intervals for definite energy allocation of any IPPCo.

\subsection{Uncertainty Model}

A general configuration is used in uncertainty set which is based on the information regarding constraint of interest (Ben-Haim, 2006). The uncertain input parameter $u$ has uncertainty $U$ defined as unconfined clique of nested sets, bounded by the uncertainty Parameter $\gamma$ for estimated $\tilde{u}$. The deviation in estimates and degree of uncertainty in different securities can be assessed by using the Ellipsoid-bound Model (EBM) of information gap uncertainty. Mathematically it should be represented as (Ben-Haim, 2006):

$$
U(\gamma, \tilde{u})=\left\{u=\tilde{u}+\Delta u: \Delta u^{\mathrm{T}} D^{-1} \Delta u \leq \gamma^{2}\right\}, \gamma \geq 0
$$

where, covariance matrix among various assets has been represented by $D$. Figure 1 shows an ellipsoid centered at $\tilde{u}$ as defined by the quadratic term. $\gamma$ is a free uncertainty parameter and represents the size of each ellipsoid. $U(\gamma, \tilde{u})$ is a region comprising of $u$, for a particular $\gamma$ and it represents cover for uncertainty.

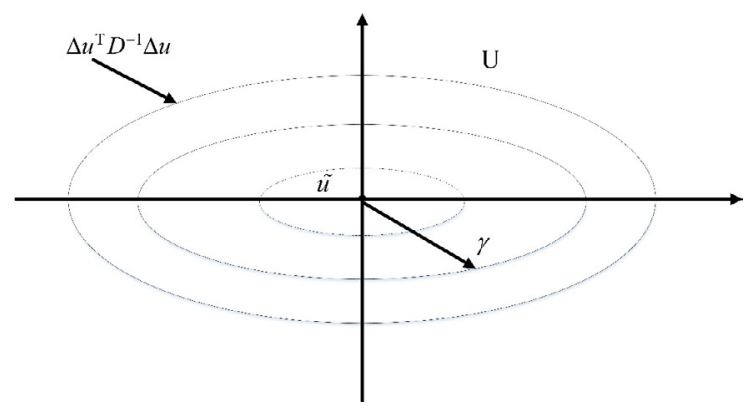

Figure 1. IGD model of uncertainty illustrating unbounded uncertainty as nested sets

\subsection{Performance Constraints}

The performance constraints express consequences to facilitate a decision maker who demands or seeks to choose the optimal solution (Ben-Haim, 2001). Uncertainty may be ruinous or favorable, subject to its risk aversion or nature that seeks risk as preferred by the decision maker. Robustness and opportuneness functions are used to meet perilous requirements and seek windfall objective for significant outcome respectively (Ben-Haim, 2001). Likelihood of fortifying windfall benefit is identified by opportuneness function, while a confident profit is assured by the robustness function. Uncertainty parameter $\gamma$ has been optimized by both functions as:

$$
\begin{aligned}
& A\left(v, p_{c}\right)=\max \left\{\gamma: \min E(v, u)>p_{c}\right\} \\
& B\left(v, p_{w}\right)=\min \left\{\gamma: \max E(v, u)>p_{w}\right\}
\end{aligned}
$$

The maximum uncertainty $\gamma$ is expressed by the robustness function $A(\mathrm{v}, \mathrm{u})$ in (2), which shows that the minimum requirement is always fulfilled i.e. critical profit, $p_{c}$ should always be less than minimum profit. So, if market falls, certain decisions should be protected as it depicts risk averting nature of a decision maker. Moreover, vision of an optimistic decision taker for taking advantage of favorable market changes can be achieved by opportunity function. To attain windfall profit as immense as windfall profit, $p_{w}$, the minimum measure of uncertainty that have to be endured is represented by opportuneness function $B(v, u)$. Whereas, uncertainty parameter $\gamma$ is assessed by both robustness function $A(\mathrm{v}, \mathrm{u})$ and opportuneness function as expressed in (2) and (3) to gain necessary results.

\section{Formulation}

This paper presents a formulation based on IGD theory for the optimization of the IPPCo's power portfolio. Real time trading strategies, i.e. bilateral and pool contract markets are contemplated in this work using location marginal pricing (LMP) scheme. Bilateral deals are contracted by IPPCo with customers located in same or different locations. Congestion may affect bilateral contracts in different locations (Menniti et al., 2007). LMP separation is caused between different locations due to congestion. For underlying contract, congestion charges are 
applicable if there is difference between LMPs located at two different positions. Depending upon the market law, the suppliers or consumers are charged respectively.

It's too hard to predict LMP's at the time of planning as they are based on real time network conditions. Therefore, LMPs based contracts are unclear and for such contracts profits can only be projected through planning. The profits are associated with each other up to a certain degree of numerous uncertain contracts offered to IPPCo.. Terms of bilateral contract (time, price, quantity etc.) are supposed and it is assumed that they are known to decision maker. Profits are considered deterministic because they are from bilateral contracts and are not affected by network constraints for being located within home location. This problem of selecting a portfolio for multiasset energy allocation is solved by formulated an IPPCo price approach with an IGD theory framework to cope with the crucial uncertainty of profits from various trading possibilities. It is supposed that markets are competent, competitive and adequately liquid for presented formulation. To represent pool, the present work takes into account day-ahead market, whereas, other trading contracts and markets can also be integrated through extension in the presented model.

It is assumed that one bilateral contract only can be made with client of a certain locality by IPPCo for simplicity in calculation and resulted in existence of a single spot market. Total of $n+1$ contracts can be made by IPPCo for considered $n$ locations; home location consumer will get one bilateral contract and bilateral contracts of non-home location clients $n-1$ and spot market contract.

\subsection{Contract price modelling}

It is supposed that a IPPCo is located at position 1. As transmission charges are not valid for user of home locality $(\mathrm{k}=0)$, contract cost would be equivalent to consensually defined cost $\eta_{1, m}^{A}$, where, $\eta_{1, m}^{A}$ the bilaterally agreed price for local consumers in $m$ th trading interval $(\$ / \mathrm{MWh})$ :

$\eta_{0, m}=\eta_{1, m}^{A} \quad \forall m$

During trading of spot market $(k=1)$, LMP would be paid to IPPCo in its own locality, where power is been inserted to the system through generators at Location 1 as defined in (5). Where, $\eta_{1, m}$ is the price of trading contract in $m$ th trading interval ( $\$ / M W h)$. Contract price for them would be:

$\eta_{1, m}=\mathrm{LMP}_{1, m} \quad \forall m$

For non-home locations bilateral contracts are represented as $k=2 \sim n$. Congestion charges applicable per unit from location 1 to location 2 for transmitting energy, at certain time $m$, would be

Congestion Charges $=\mathrm{LMP}_{2, m}-\mathrm{LMP}_{1, m} \quad \forall m$

As per market rule, based on $\beta(0 \leq \beta \leq 1)$, congestion charges are paid by contract-holders uniformly on such contracts. For IPPCo, the actual contract prices are

$\eta_{k, m}=\eta_{k, m}^{A}-\beta\left(\mathrm{LMP}_{2, m}-\mathrm{LMP}_{1, m}\right) \quad \forall m$

For $k=2 \sim n$

Therefore, all contracts rely upon persistently uncertain LMPs, apart from local bilateral contract $(k=0)$, due to which contract prices become uncertain. To evaluate overall portfolio profit, cost of several trading choices are considered and profits regarding each trading option was calculated.

\subsection{Portfolio profit}

Weighted summation of individual profits from each trade is overall portfolio profit $E_{p}$. Local bilateral contract's profit is indexed as zero. Portfolio's future net profit with relevant weight $w_{k}$ of $n+1$ assets and profits $r_{k}$ is

$E_{p}=\sum_{k=0}^{n} w_{k} p_{k}$

s.t. $\quad \sum_{k=0}^{n} w_{k}=1$

and $w_{k} \geq 0$

Any asset's profit can be represented as:

Profit $=\frac{\text { Revnue }- \text { Cost }}{\text { Cost }}$

By trading $t_{k, m}$ power at contract price $\eta_{k, m}$ in each option generated revenue for each trading interval can be calculated as

$E V\left(\mathrm{t}_{k, m}, \eta_{k, m}\right)=\mathrm{Tt}_{k, m} \eta_{k, m}$

http://www.sic.ici.ro 
A quadratic cost curve

$C\left(t_{k, m}, \eta_{m}^{F}\right)=\left(x+y t_{k, m}+z t_{k, m}^{2}\right) T \eta_{m}^{F}$ can be used to represent generation cost which consists of variable and permanent cost, where $t_{m}$ is generation outcome (MW) and T represents the duration of trading intervals in hours.

Generated power at each trading interval is then allocated among $n+1$ trading options. Thus, generation cost for $t_{m}$ is divided for power traded in each option. Share of cost for power traded in $k$ th contract $t_{k, m}$, at $k$ th trading interval can be calculated as

$$
C\left(\mathrm{t}_{k, m}, \eta_{m}^{F}\right)=\mathrm{T} y_{m} \eta_{m}^{F} \mathrm{t}_{k, m}
$$

where fuel prices and $y_{m}=\left(x+y t_{m}+z t_{m}^{2}\right) /\left(t_{m}\right)$ were supposed already determined for the planning period needs to be considered.

For trading period $\mathrm{H}$, profit from every trading contract, $k(k=1 \sim n)$, can be calculated using (12) and (13) and averaging out the profits of each trading interval $m$ as

$$
\begin{aligned}
& p_{k}=\frac{1}{H} \sum_{m=1}^{H} \frac{T\left(\eta_{k, m}-y_{m} \eta_{m}^{F}\right) t_{k, m}}{T y_{m} \eta_{m}^{F} t_{k, m}} \\
& \Rightarrow p_{k}=\frac{1}{H} \sum_{m=1}^{H}\left(\frac{\eta_{k, m}}{J_{m}}-1\right)
\end{aligned}
$$

where $J_{m}=y_{m} \eta_{m}^{F}$

\subsection{IGD Theory formulation}

The problem of portfolio optimization discussed above is grounded on the IGD theory of section 2. Portfolio profit $E_{p}$ can be evaluated with the future profits of the trading choices $r_{k}$ and their percentage of energy distribution $w_{k}$. Decision variables are the corresponding weights. Except $p_{0}$, uncertain profits from trading options are considered as uncertain parameters of the problem. Trading schemes are developed for opportunity functions and robustness using appropriate constraints and system model, for the considered uncertainty model.

\subsubsection{System Model}

Net future portfolio profit for known (local bilateral contract profit $\left(p_{0}\right)$ and unknown $k=1 \sim n$ contract profits) assets is supposed as
$E_{p}(w, p)=w_{0} p_{0}+\sum_{k=1}^{n} w_{k} p_{k}$

If $p=\left[p_{1} p_{2} \ldots p_{n}\right]$ and $w=\left[w_{1} w_{2} \ldots w_{n}\right],(16)$ can be written as

$$
E_{p}(w, p)=w_{0} p_{0}+w^{T} p
$$

$\Delta p$ can be deviated from its expected value $\tilde{p}$ because $p$ is uncertain

$p=\tilde{p}+\Delta p$

where $\Delta p=\left[\Delta p_{1} \Delta p_{2} \ldots \Delta p_{n}\right]$ an $\mathrm{d} \tilde{p}=\left[\tilde{p}_{1} \tilde{p}_{2} \ldots \tilde{p}_{n}\right]$

Variation in contract prices $\Delta \eta_{k, m}$ from their expected value $\tilde{\eta}_{k, m}$ causes variation in profits. The discussed relation can be stated as

$p_{k}=\sum_{m=1}^{H} \frac{1}{J_{m}}\left(\tilde{\eta}_{k, m}+\Delta \eta_{k, m}\right)-1$

The portfolio profit or system model can be obtained as:

$$
E_{p}(w, p)=w_{0} p_{0}+w^{\mathrm{T}}(\tilde{p}+\Delta p)
$$

\subsubsection{Uncertainty Model}

Information regarding the unit of fluctuation in costs of numerous trading choices and their correlated or anti-correlated deviations is shown by a distinctive covariance matrix for various asset profits $k=1 \sim n$. A model of IGD Theory which is ellipsoid bound and uses the above information to formulate the uncertainty of profits, by creating matrix of uncertainty shape. It is possible to use this model with only diagonal matrix elements without any correlation between securities. Uncertainty model can be based on the available information as:

$$
\begin{aligned}
& U(\gamma, \tilde{p})=\left\{p: p+\tilde{p}+\Delta p: \Delta p^{\mathrm{T}} D^{-1} \Delta p \leq \gamma^{2}\right\} \\
& \gamma \geq 0
\end{aligned}
$$

Here $n \times n$ is the size of $D$ and number of uncertain trades are represented by $n . D$ is a positive definite matrix with real and symmetric characteristics. (14) and (15) can be used to calculate matrix elements

$$
D_{k, l}=\operatorname{cov}\left(p_{k}, p_{l}\right)
$$

Evaluation of covariance matrices has been done between uncertain profits. For planning period $H$, elements of $D$ are obtained by averaging 
out the covariance amid these profits which are assessed for each trading interval.

$D_{k, l}=\frac{1}{H} \sum_{m=1}^{H} \frac{\operatorname{cov}\left(\eta_{k, m}, \eta_{l, m}\right)}{J_{m}^{2}}$

For $k \in\{1,2, \ldots, \mathrm{n}\}, 1 \in\{1,2, \ldots, \mathrm{n}\}$

\subsubsection{Robustness Function}

Every IPPCo having risk-aversion nature always try to protect itself from uncertain expenditures. In case of a certain decline in market profits, the robustness function estimates the degree of decline and ensure the critical profit $p_{c}$ should be equal or less than minimum profit or least profit should not be less than a critical profit $p_{c}$. Degree of uncertainty that any choice can tolerate is evaluated by this function without any performance requirements sacrifice. For portfolio selection strategy $w$, robustness is uncertainty parameter $\gamma$ largest value, for critical profit $p_{c}$, such that only $E_{p}(w, r)$ is outcome for any profit in region $U(\gamma, \tilde{p})$ which is not less than $p_{c}$. To evaluate performance, the requirement specified in (2) should be satisfied, when all $p \in U(\gamma, \tilde{p})$, for uncertainty $\gamma$, portfolio profit's minimum value would be:

$$
\begin{aligned}
& \min _{\Delta p} E_{p}(w, p)=w_{0} p_{0}+w^{\mathrm{T}} \tilde{p}+w^{\mathrm{T}} \Delta p \\
& \text { s.t. } \Delta p^{\mathrm{T}} D^{-1} \Delta p \leq \gamma^{2}
\end{aligned}
$$

We can rewrite above equations as

$$
\begin{aligned}
& \min _{\Delta p} E_{p}(w, p)=w_{0} p_{0}+w^{\mathrm{T}} \tilde{p} \\
& +\min _{\Delta p}\left\{w^{\mathrm{T}} \Delta p: \Delta p^{\mathrm{T}} D^{-1} \Delta p \leq \gamma^{2}\right\}
\end{aligned}
$$

Lagrange relaxation method can be used to solve above optimization problem and it gives

$$
\Delta p= \pm \gamma \frac{D w}{\sqrt{w^{\mathrm{T}} D w}}
$$

Negative value of $\Delta p$ is used $(\gamma>0)$ for minimum $E_{p}(w, \gamma)$, which gives

$$
\min E_{p}(w, \gamma)=w_{0} p_{0}+w^{\mathrm{T}} \tilde{p}-\gamma \sqrt{w^{\mathrm{T}} D w}
$$

$p_{c}$ should be equal to minimum portfolio profit, specified as:

$$
\begin{aligned}
& w_{0} p_{0}+w^{\mathrm{T}} \tilde{p}-\gamma \sqrt{w^{\mathrm{T}} D w}=p_{c} \\
& \Rightarrow \gamma\left(p_{c}\right)=\frac{w_{0} p_{0}+w^{\mathrm{T}} \tilde{p}-p_{c}}{\sqrt{w^{\mathrm{T}} D w}}
\end{aligned}
$$

For a targeted profit $p_{c}$, the largest value of $\gamma$ is represented by robustness function:

$A\left(w, p_{c}\right)=\max _{w} \gamma\left(p_{c}\right)=\max _{w} \frac{w_{0} p_{0}+w^{\mathrm{T}} \tilde{p}-p_{c}}{\sqrt{w^{\mathrm{T}} D w}}$

As $\gamma$ represents gap from the nominal estimate and it is the size of uncertain ellipsoid, therefore, its value can never be negative. So, it is zero for

$p_{c}>\left(w_{0} p_{0}+w^{\mathrm{T}} \tilde{p}\right)$.

Optimal energy allocation strategy for portfolio optimization is dependent on uncertainty of assets and profits in addition to tendency of deviation. Increase in targeted critical profits results in decreasing robustness. For IPPCo's energy distribution, optimal weights for maximizing robustnes $A\left(w, p_{c}\right)$ are selected to maximize the portfolio profit, while $w^{\mathrm{T}} D w$ would be minimized, with respect to other constrictions.

\subsubsection{Opportuneness Function}

An IPPCo has to face definite uncertainty to get advantage from chances of extreme market prices. To get possible reward as large as $p_{w}$, opportunity $B\left(w, p_{c}\right)$ is the lowest uncertainty level which has to be endured. When all $p \in U(\gamma, \tilde{p})$, for $\gamma>0$ subject to (21), maximum possible profit up to uncertainty $\gamma$, Lagrange method can be used to calculate it while considering positive value of $\Delta p$ from (27):

$\max _{\Delta p} E_{p}(w, p)=w_{0} p_{0}+w^{\mathrm{T}} \tilde{p}+\gamma \sqrt{w^{\mathrm{T}} D w}$

$E_{p}(w, \gamma)$ should have its maximum value large enough to get equal to windfall profit $p_{w}$,

Therefore

$$
\begin{aligned}
& w_{0} p_{0}+w^{\mathrm{T}} \tilde{p}+\gamma \sqrt{w^{\mathrm{T}} D w}=p_{w} \\
& \Rightarrow \gamma\left(p_{w}\right)=\frac{p_{w}-\left(w_{0} p_{0}+w^{\mathrm{T}} \tilde{p}\right)}{\sqrt{w^{\mathrm{T}} D w}}
\end{aligned}
$$


Least possible uncertainty is been represented above based on (3). So, opportunity function can be evaluated using (34) as

$$
B\left(w, p_{w}\right)=\min _{w} \gamma\left(p_{w}\right)=\min _{w} \frac{p_{w}-\left(w_{0} p_{0}+w^{\mathrm{T}} \tilde{p}\right)}{\sqrt{w^{T} D w}}
$$

In (35), $p_{w}>\left(w_{0} p_{0}+w^{\mathrm{T}} \tilde{p}\right)$, else it would be equal to zero. If Windfall profits increases, opportunity function $B\left(w, p_{w}\right)$ also increases. Opportunity function $B\left(w, p_{c}\right)$ is minimized by maximizing portfolio profit and $w^{T} D w$ for a certain value of $p_{w}$.

Ultimately as per IPPCo's nature, trading strategy $w$ is decided according to (31) and (35) for multiple values of $p_{c}$ and $p_{w}$. When $w^{T} D w$ is to be minimized, $A\left(w, p_{c}\right)$ is maximized, whereas, $w^{T} D w$ is to be maximized when $B\left(w, p_{c}\right)$ is minimized. Therefore, portfolio optimization strategies are divergent which are offered by two optimization problems. An opportunistic approach is commonly selected with greater uncertainty contracts and less robustness by an optimistic decision maker. The limiting constraint in this problem provides that the user must not be located in home locality on trading contract is:

$\stackrel{\text { Min }}{t_{k, m}} s_{k, m} \leq t_{k, m} \leq t_{k, m}^{\operatorname{Max}} s_{k, m}$ for $\forall k, \forall m$ and

$(i \neq 1)$

$s_{k, m} \in\{0,1\}$ for $\forall k, \forall m$ and $(i \neq 1)$

where selection of contract is decided by variable $s_{k, m}$. (31) and (35) are MINLP problems each under the constraints (5), (6), (36) and (37). To attain asset weights $w$, these problems can be solved.

\section{Results and case study}

A PJM market real time scenario is considered to analyze the suggested portfolio optimization method for IPPCo [18]. IPPCo with generation specifications $x=695.317 \mathrm{MBtu}, y=7.97 \mathrm{MBtu} /$ MW and $z=0.00119 \mathrm{MBtu} / \mathrm{MW}$ of total 500 MW total capacity, aims to organize its trading scheme for April 2018. Trading interval is one hour and one month is the considered planning period with total of $30 \times 24=720$ trading intervals. Stable fuel prices are considered for all trading interval at $4 \$$ MBtu. As shown in Table 1, IPPCo transacts its scheduled generation (supposed to be full capability) among multiple bilateral contracts according to relative contract specifications with six different locations and in day-ahead spot market.

Home location for trading IPPCo is considered to be APC and the indexed as Contract $0(i=0)$ is the bilateral contract with it. Spot contract price for IPPCo are the LMPs of APC and indexed as Contract $1(i=1)$.

Hourly day ahead LMPs of month April, from year 2011 to 2017 , have been used to calculate the expected/forecasted LMPs. These calculate expected contract prices $\tilde{\eta}_{k, m}$ instituted on the conditions presented in Table 1 using (4)-(7), and cogitating $\gamma=1$. Amongst the entire $n+1$ contracts, projected prices $\tilde{p}$ are approximated using expected value of contract prices $\tilde{\eta}_{k, m}$ (Contracts 1-6), for all profits except Contract 0 and generation requirement is grounded on relation presented in (19).

Table 1. Bilateral contracts specifications

\begin{tabular}{ccccc}
\hline $\begin{array}{c}\text { Contract } \\
\text { Number }\end{array}$ & $\begin{array}{c}\text { Location } \\
\text { Name }\end{array}$ & $\begin{array}{c}\text { Contract Prices } \\
(\$ / \mathrm{MWh})\end{array}$ & $\begin{array}{c}\text { Min. } \\
(\mathrm{MW})\end{array}$ & $\begin{array}{c}\text { Max. } \\
(\mathrm{MW})\end{array}$ \\
\hline 0 & APC & 51.5 & 50 & 350 \\
2 & PECO & 54.0 & 50 & 350 \\
3 & AEP & 43.5 & 50 & 350 \\
4 & PENELEC & 49.5 & 50 & 350 \\
5 & AECO & 56.0 & 50 & 350 \\
6 & COMED & 42.0 & 50 & 350 \\
\hline
\end{tabular}

With appropriate function in MATLAB ${ }^{\circledR}$, uncertainty shape matrices are calculated from (23) for each trading interval using variancecovariance between uncertain contract prices. There are 720 matrices of $6 \times 6$ order for the considered data and due to space limitation, all matrices are not demonstrated in this paper.

Table 2. Uncertainty shape matrix among profits

\begin{tabular}{ccccccc}
\hline Contract & 1 & 2 & 3 & 4 & 5 & 6 \\
\hline 1 & 1.022 & 0.656 & 0.651 & 0.401 & 0.405 & 0.187 \\
2 & 0.655 & 0.365 & 0.355 & 0.276 & 0.279 & 0.121 \\
3 & 0.660 & 0.364 & 0.359 & 0.249 & 0.248 & 0.117 \\
4 & 0.397 & 0.265 & 0.261 & 0.199 & 0.185 & 0.095 \\
5 & 0.266 & 0.248 & 0.242 & 0.178 & 0.201 & 0.088 \\
6 & 0.193 & 0.109 & 0.110 & 0.087 & 0.081 & 0.082 \\
\hline
\end{tabular}

Variability and co-variability of profits is represented by uncertainty shape matrix for entire period of planning in Table 2. Individual 
variability is represented by diagonal values, whereas, co-variability amid profits of uncertain trades is shown by off-diagonal elements. Highest variability is represented by Contract 1 , however with minimum for Contract 6 , other contracts have relatively less variability.

\subsection{Simulations}

Maximum value of portfolio profit $\tilde{E}_{p}(w, \tilde{p})$ is evaluated by optimizing (20), for estimated values of input parameters and without considering any uncertainty, (i.e., $\Delta p=0$ ), subject to (5), (6), (36) and (37). Critical profit goals $p_{c}$ are anticipated below portfolio profit $\tilde{E}_{p}(w, \tilde{p})$ in diminishing small steps for robust decision making. However, windfall profits are deemed above $\tilde{E}_{p}(w, \tilde{p})$ in cumulative small stages for opportunistic strategies. Optimization is executed for robustness (31) and opportunity (35) for all $p_{w}$ greater and all $p_{c}$ lesser than the portfolio profit $\tilde{E}_{p}(w, \tilde{p})$ individually, dependent on (5), (6), (36) and (37).

In terms of weight $w$, specific trading scheme is acquired for each value of $p_{c}$ and $p_{w} .6706$ real and 5208 discrete variables are considered to resolve each optimization problem in current analysis, by GAMS solver COUENNE 0.5@ using high profile desktop computer, with 2.6 seconds as the average solution time.

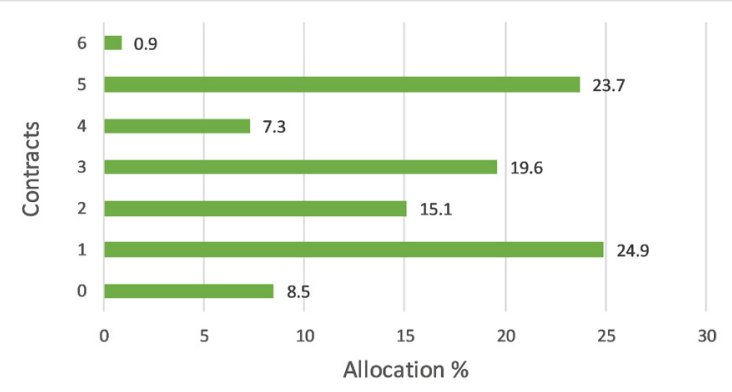

Figure 2. Distribution of energy without consideration of uncertainty

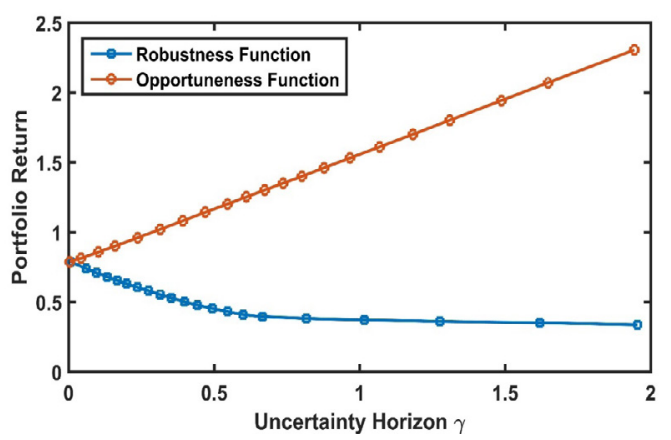

Figure 3. Portfolio profit for various uncertainty values

\subsection{Results}

The maximum obtained portfolio profit $\tilde{E}_{p}(w, \tilde{p})$ corresponding to energy allocation $w$ in Figure 2 for zero uncertainty is 0.7744 . Therefore IPPCo imitates risk neutral attitude after it considers projected value as accurate value for taking decision and without considering any future uncertainty. Both opportunity and robustness are zero at $p_{c}=p_{w}=0.7744$. As shown in Figure 3, with variation of profit target from 0.7744 , values for two functions increase in different directions. Estimations are correct in this condition, consequently expected profits are equal to targeted profits.

For different values of $p_{c}$ and $p_{w}$, uncertainty/ error $\gamma$ from expectation is calculated by two optimization problems (31) and (35). Targeted profits $p_{c}$ and $p_{w}$ versus horizon of uncertainty assessed for robustness and opportunity functions is shown in Figure 3. Considering two faces of uncertainty, this replicates performance quantification in various uncertainty degrees. Robustness function calculates a certain tolerable deviation to guarantee $p_{c}$. Whereas opportunity of fortifying profits up to $p_{w}$ is provided by same level of uncertainty, but there is no guarantee to achieve those profits. Any strategy can be opted by a decision maker according to his understanding about market and its nature for selecting portfolio. A decision maker mostly chooses a strategy to secure profit $p_{c}$ in case of uncertainty $\gamma$ if he owns risk averting nature. However, if he is a risk loving decision maker, he can choose a strategy with uncertainty and it can lead to achieve a profit as large as $p_{w}$.

\subsubsection{Robust Portfolio Selection}

According to optimization results shown in Figures 3 to Figure 5, the critical profit $p_{c}$ ranges between 0.7744 to 0.3474 . As shown in Figure 3 the achieved robustness at $p_{c}=0.7744$, i.e. as $p_{c}$ decreases from 0.7744 to 0.3474 , the permissible inaccuracy is zero and fluctuates from zero to 1.955. Robustness of the choice declines with the rising values of higher profit targets as they are more demanding as shown in Table 3. The robustness signifies allowable range of $\gamma$ for each targeted profit $p_{c}$, so that in case of unfavorable variation in the market profits, the decision can give profit no less than $p_{c}$. For instance, the obtained robustness at $p_{c}=0.6$ is $A(w, 0.6)=0.2392$

http://www.sic.ici.ro 
Table 3. Portfolio profits exercising robustness approach for various disparaging price movements centered on $A$

\begin{tabular}{cccccccccccc}
\hline & $\mathbf{0 . 0 0 0 0}$ & $\mathbf{0 . 1 0 3 5}$ & $\mathbf{0 . 1 9 7 2}$ & $\mathbf{0 . 2 3 9 2}$ & $\mathbf{0 . 3 1 3 7}$ & $\mathbf{0 . 3 9 5 3}$ & $\mathbf{0 . 4 3 0 7}$ & $\mathbf{0 . 6 1 9 6}$ & $\mathbf{0 . 6 9 4 6}$ & $\mathbf{0 . 8 2 8 8}$ & $\mathbf{1 . 9 5 5}$ \\
\hline $\mathbf{0 . 8 0}$ & 0.7744 & 0.7744 & 0.6611 & 0.6443 & 0.6006 & 0.5541 & 0.5326 & 0.4227 & 0.3844 & 0.3080 & -0.3360 \\
$\mathbf{0 . 7 1}$ & 0.7769 & 0.7194 & 0.6625 & 0.6391 & 0.6006 & 0.5597 & 0.5329 & 0.4253 & 0.3884 & 0.3135 & -0.3173 \\
$\mathbf{0 . 6 4}$ & 0.7410 & 0.6826 & 0.6348 & 0.6037 & 0.5702 & 0.5199 & 0.5089 & 0.4027 & 0.3611 & 0.2900 & -0.3301 \\
$\mathbf{0 . 6 1}$ & 0.7291 & 0.6816 & 0.6287 & 0.6034 & 0.5614 & 0.5175 & 0.4994 & 0.4070 & 0.3633 & 0.2943 & -0.2999 \\
$\mathbf{0 . 5 5}$ & 0.7481 & 0.6977 & 0.6497 & 0.6305 & 0.5925 & 0.5576 & 0.5361 & 0.4469 & 0.4109 & 0.3424 & -0.2210 \\
$\mathbf{0 . 5}$ & 0.7314 & 0.6851 & 0.6418 & 0.6231 & 0.5882 & 0.5486 & 0.5368 & 0.4512 & 0.4081 & 0.3511 & -0.1734 \\
$\mathbf{0 . 4 8}$ & 0.6514 & 0.6221 & 0.5832 & 0.5750 & 0.5427 & 0.5162 & 0.5114 & 0.4375 & 0.4145 & 0.3734 & -0.0238 \\
$\mathbf{0 . 4 3}$ & 0.6051 & 0.5707 & 0.5463 & 0.5398 & 0.5104 & 0.4888 & 0.4812 & 0.4296 & 0.4049 & 0.3775 & 0.0637 \\
$\mathbf{0 . 3 9}$ & 0.5500 & 0.5353 & 0.5107 & 0.5030 & 0.4862 & 0.4659 & 0.4591 & 0.4147 & 0.3978 & 0.3721 & 0.1197 \\
$\mathbf{0 . 3 7}$ & 0.4696 & 0.4541 & 0.4456 & 0.4356 & 0.4333 & 0.4201 & 0.4162 & 0.3878 & 0.3852 & 0.3685 & 0.2301 \\
$\mathbf{0 . 3 5}$ & 0.4022 & 0.3947 & 0.3875 & 0.3848 & 0.3725 & 0.3721 & 0.3663 & 0.3540 & 0.3561 & 0.3453 & 0.3474 \\
\hline
\end{tabular}

which epitomizes that for securing a minimum portfolio the allowable uncertainty/error is 0.2392 for 0.6. As shown in Figure 4, with decrease in targeted profits, the expected portfolio profit $E\left(w\left(A\left(p_{c}\right)\right), \tilde{p}\right)$ also reduces but it always stays higher than the equivalent targeted value. The tiff between maximum portfolio profit $\tilde{E}_{p}(w, \tilde{p})$ and expected portfolio profit $E\left(w\left(A\left(p_{c}\right)\right), \tilde{p}\right)$ for a certain value of $p_{c}$ is the cost has to be endured by IPPCo for robustness of selected decision.

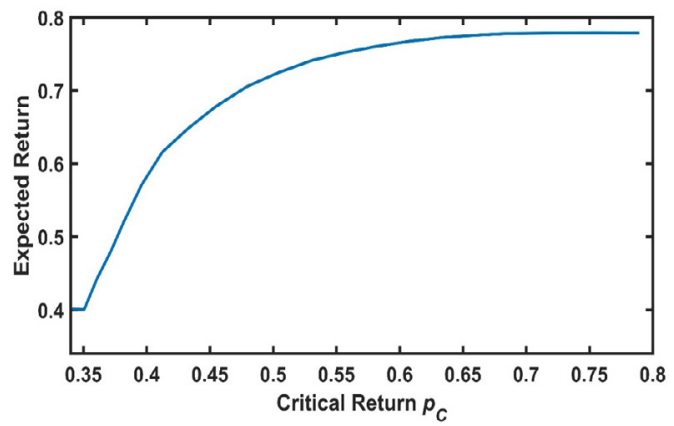

Figure 4. Expected profit for different critically targeted profits

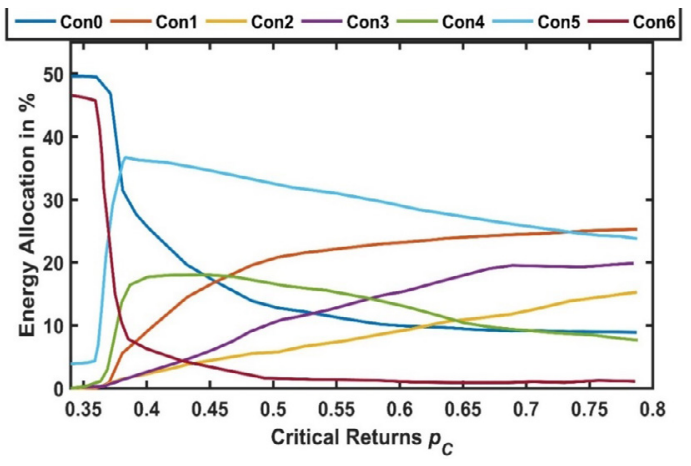

Figure 5. Energy distribution in various contracts for optimal critical profits

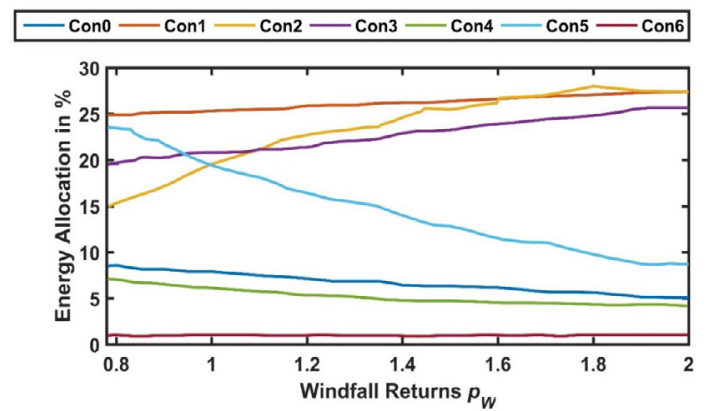

Figure 6. Energy distribution in various contracts for optimal windfall profits

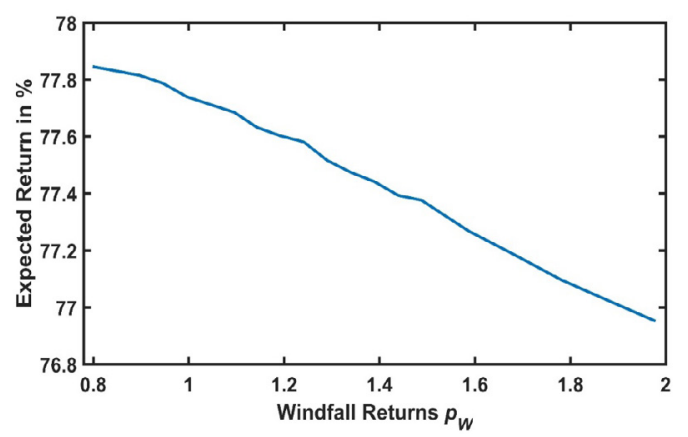

Figure 7. Anticipated portfolio profits for diverse windfall profits

\subsubsection{Opportunistic Portfolio Selection}

This selection represents the risk pursuing attitude of a decision maker in way of favorable face of uncertainty. This reflects that there may be an opportunity for securing windfall profits in uncertainty. Figures 3, 6 and 7 signifies the results of optimization for opportuneness $B\left(w, p_{w}\right)$ (35) varying from 0 to 1.73 for windfall profits $p_{w}$. As shown in Figure 3, there is a increase from 0 to 1.955 in $B\left(w, p_{w}=0.9\right)=0.158$ with windfall 
Table 4. Portfolio profits exercising opportunity approach for various disparaging price movements centered on $B$

\begin{tabular}{ccccccccccc}
\hline & $\mathbf{0 . 0 0 0 0}$ & $\mathbf{0 . 0 9 3 6}$ & $\mathbf{0 . 2 8 8 4}$ & $\mathbf{0 . 4 8 2 0}$ & $\mathbf{0 . 6 1 0 4}$ & $\mathbf{0 . 6 7 4 7}$ & $\mathbf{0 . 9 3 0 5}$ & $\mathbf{1 . 1 8 5 5}$ & $\mathbf{1 . 4 3 9 6}$ & $\mathbf{1 . 5 6 6 9}$ \\
\hline $\mathbf{0 . 8}$ & 0.7781 & 0.8294 & 0.9399 & 1.0572 & 1.1258 & 1.1589 & 1.3087 & 1.4546 & 1.5963 & 1.6734 \\
$\mathbf{0 . 8 5}$ & 0.7759 & 0.8273 & 0.9411 & 1.0519 & 1.1310 & 1.1630 & 1.3179 & 1.4605 & 1.6059 & 1.6837 \\
$\mathbf{1 . 0}$ & 0.7786 & 0.8302 & 0.9464 & 1.0568 & 1.1313 & 1.1756 & 1.3250 & 1.4742 & 1.6207 & 1.6917 \\
$\mathbf{1 . 1 5}$ & 0.7798 & 0.8323 & 0.9451 & 1.0618 & 1.1410 & 1.1717 & 1.3244 & 1.4771 & 1.6304 & 1.7060 \\
$\mathbf{1 . 2 5}$ & 0.7743 & 0.8301 & 0.9461 & 0.1017 & 1.1423 & 1.1748 & 1.3327 & 1.4790 & 1.6304 & 1.7091 \\
$\mathbf{1 . 3}$ & 0.7749 & 0.8341 & 0.9422 & 1.0521 & 1.1306 & 1.1663 & 1.3093 & 1.4573 & 1.6071 & 1.6815 \\
$\mathbf{1 . 5}$ & 0.7788 & 0.8335 & 0.9446 & 1.0623 & 1.1453 & 1.1810 & 1.3312 & 1.4938 & 1.6467 & 1.7172 \\
$\mathbf{1 . 7}$ & 0.7695 & 0.8245 & 0.9515 & 1.0672 & 1.1394 & 1.1873 & 1.3420 & 1.4991 & 1.6542 & 1.7240 \\
$\mathbf{1 . 9}$ & 0.7752 & 0.8293 & 0.9503 & 1.0652 & 1.1466 & 1.1885 & 1.3480 & 1.4969 & 1.6588 & 1.7368 \\
$\mathbf{2 . 0}$ & 0.7746 & 0.8269 & 0.9503 & 1.0676 & 1.1467 & 1.1889 & 1.3416 & 1.5007 & 1.6593 & 1.7351 \\
\hline & & & & & & & & & &
\end{tabular}

profits. This implies that with increase in uncertainty, there is more opportunity of securing windfall benefits as shown in Table 4. Minimum uncertainty given by $B\left(w, p_{w}\right)$ has to be tolerated by IPPCo to accomplish profits as huge as $p_{w}$. Hence, freedom to decision towards uncertainty reduces, if a IPPCo wishes greater windfall profits. It can be explained in a simple way, for example, $B\left(w, p_{w}=0.9\right)=0.158$ indicates that for the market profits increasing up to 0.158 degree, IPPCo can accomplish profit up to 1 from its trading portfolio. As shown in Figure 6, with increase in aspiration for windfall profits, there is rise in energy allocation for trades with greater variability and vice-versa. This occurs as there is stronger likelihood of promising price spikes for contracts with higher variability. Due to higher variability, there is increase in allocation for Contracts 1,2 and 3 , whereas low variability Contracts 0,4 and 5 faces decrease in allocation. However, owing to stumpy profit and stumpy variance, allocation in Contract 6 stays insignificant.

A bargain is created between opportunity and windfall due to growing aspiration for windfall profits. It can be illustrated as: receiving higher uncertainty also escort windfall profits, this resulted in decreased possibility of taking advantage of the opportunity arose owing to positive uncertainty. Figure 7 indicates that expected profits $E\left(w\left(B\left(p_{w}\right)\right), \tilde{p}\right)$ are lesser than windfall profits $E\left(w\left(B\left(p_{w}\right)\right), \tilde{p}\right)$ is the portfolio profit and it will be up to its expected value if price spikes do not occur due to selection of opportunistic strategy. Any difference between maximum profit and expected profit is the price of consenting likelihood of greater anticipated advantage, which have to be tolerated by a IPPCo if there is no favorable change in market prices according to its desire. Therefore, the outcomes clearly emphasize that higher variability contracts increase the opportunity of higher benefits, on the other hand, greater allocation in low variability contracts increases the robustness of the decision. Consequently, there exists a trade-off between opportuneness and robustness; extremely robust decision will be least opportunistic and if any particular decision is extremely opportunistic then it will not be robust.

\section{Conclusion}

This paper considers the trading portfolio optimization of IPPCo comprising of congestion and pool price uncertainties in pool and bilateral markets. Severe uncertainty is troublesome and found complicated to be tackled by implementing the traditional decision making strategies as they are decided by considering the estimated market profits that usually vary as compared to the actual ones. By computing the deviation between estimated and actual values, an IGD theory has been acquired to deal with this uncertainty. Decisions presented by this formulation are opportunistic towards seizing higher gains and robust towards losses, paralleled to conventional portfolio theory.

This proposed framework has been substantiated by assuming deviations in profit estimations. With the help of real time scenario of PJM market, the results indicate that the proposed method can promise portfolio profit when price changes in an unfavorable manner within robustness region. 
Similarly, this allows an IPPCo to realize windfall profits triggered owing to promising price spikes. A variety of decisions is offered to IPPCo for selection, as revealed by results. For optimal portfolio selection, these decisions are assessed for various conditions, such as trade-offs present between robustness and opportuneness, reward and robustness, and opportunity and windfall gain. But there is some cost which has to be

\section{REFERENCES}

1. Aien, M., Hajebrahimi, A. \& FotuhiFiruzabad, M. (2016). A comprehensive review on uncertainty modeling techniques in power system studies, Renewable and Sustainable Energy Reviews, 57, 1077-1089.

2. Ben-Haim, Y. (2001, June). Decision tradeoffs under severe info-gap uncertainty. In 2nd International Symposium on Imprecise Probabilities and Their Applications, Ithaca, New York (pp. 32-39).

3. Ben-Haim, Y. (2006). Info-gap decision theory: decisions under severe uncertainty. Elsevier.

4. Carlsson, C. \& Fullér, R. (2003). A fuzzy approach to real option valuation, Fuzzy sets and systems, 139(2), 297-312.

5. Dagoumas, A. S., Koltsaklis, N. E. \& Panapakidis, I. P. (2017). An integrated model for risk management in electricity trade, Energy, 124, 350-363.

6. Đogić, M. (2017). Growth strategies of electric utilities in context of deregulation and liberalization of electricity market, Management: journal of contemporary management issues, 22(2), 79-98.

7. Faia, R., Pinto, T., Vale, Z. \& Corchado, J. M. (2018, June). Multi-Objective Portfolio Optimization of Electricity Markets Participation. In 2018 Power Systems Computation Conference (PSCC) (pp. 1-6). IEEE.

8. Gedra, T. W. (1994). Optional forward contracts for electric power markets, IEEE Transactions on Power Systems, 9(4), 1766-1773.

9. Gökgöz, F. \& Atmaca, M. E. (2012). Financial optimization in the Turkish electricity market: endured by IPPCo to assure a minimum level of advantages, along with securing higher gains, depending on selected decision's characteristics. The presented scheme can be extended for making trading decision of IPPCo in numerous markets by considering the influence of external market uncertainties and several kinds of contracts are involved in it.

Markowitz's mean-variance approach, Renewable and Sustainable Energy Reviews, 16(1), 357-368.

10. He, G., Chen, Q., Kang, C., Pinson, P. \& Xia, Q. (2016). Optimal bidding strategy of battery storage in power markets considering performance-based regulation and battery cycle life, IEEE Transactions on Smart Grid, 7(5), 2359-2367.

11. Jalilvand-Nejad, A., Shafaei, R. \& Shahriari, H. (2017). A Genco self-scheduling problem with correlated prices using a new robust optimization approach, International Journal of Production Research, 55(11), 3249-3265.

12. Markowitz, H. (1952). Portfolio selection, The journal of finance, 7(1), 77-91.

13. Menniti, D., Musmanno, R., Scordino, N., Sorrentino, N. \& Violi, A. (2007, June). Managing price risk while bidding in a multimarket environment. In Power Engineering Society General Meeting, 2007. IEEE (pp. 1-10). IEEE.

14. Nojavan, S., Zare, K. \& Ashpazi, M. A. (2015). A hybrid approach based on IGDTMPSO method for optimal bidding strategy of price-taker generation station in day-ahead electricity market, International Journal of Electrical Power \& Energy Systems, 69, 335-343.

15. Nojavan, S., Ghesmati, H. \& Zare, K. (2016). Robust optimal offering strategy of large consumer using IGDT considering demand response programs, Electric Power Systems Research, 130, 46-58.

16. Orgaz, A., Bello, A. \& Reneses, J. (2018, June). A Monte Carlo approach to represent uncertainty in the European electricity 
market. In 2018 15th International Conference on the European Energy Market (EEM) (pp. 1-6). IEEE.

17. Shahidehpour, M., Yamin, H. \& Li, Z. (2003). Market operations in electric power systems: forecasting, scheduling, and risk management. John Wiley \& Sons.

18. Statman, M. (1987). How many stocks make a diversified portfolio?, Journal of financial and quantitative analysis, 22(3), 353-363.
19. Vatani, B., Chowdhury, B., Dehghan, S. \& Amjady, N. (2018). A critical review of robust self-scheduling for generation companies under electricity price uncertainty, International Journal of Electrical Power \& Energy Systems, 97, 428-439.

20. Zhang, H., Sun, H., Zhang, Q. \& Kong, G. (2018). Microgrid Spinning Reserve Optimization with Improved Information Gap Decision Theory, Energies, 11(9), 2347. 